\title{
THE INFLUENCE OF DIETARY PATTERNS ON ARTERIAL STIFFNESS, LIPID METABOLISM, AND LIVER AND RENAL FUNCTION IN THE POPULATION OF GREATER POLAND
}

\author{
Katarzyna Skrypnik ${ }^{1}$, Paweł Bogdanski ${ }^{2}$, Marta Kubasik², Natalia Wawrzyniak ${ }^{1}$, \\ Leszek Markuszewski , Joanna Suliburska ${ }^{1 凶}$ \\ ${ }^{1}$ Institute of Human Nutrition and Dietetics, Poznań University of Life Sciences \\ Wojska Polskiego 31, 62-624 Poznań, Poland \\ ${ }^{2}$ Department of Treatment of Obesity, Metabolic Disorders and Clinical Dietetics, Poznan University of Medical Sciences \\ Szamarzewskiego 84, 60-569 Poznań, Poland \\ ${ }^{3}$ Center for Heart and Vascular Diseases, Internal and Metabolic Diseases Mazovian Specialist Hospital, \\ Faculty of Medical Sciences and Health Sciences, University of Humanities and Technology in Radom \\ Bolesława Chrobrego 27, 26-600 Radom, Poland
}

\begin{abstract}
Background. The consumption of breakfast, salty meals, red meat, whole grain products, and dark chocolate are dietary habits that influence health, but the effects on arterial stiffness have not been well-investigated.

Aim. To assess the effects of selected dietary patterns on arterial stiffness, liver and renal function, inflammation, and glucose and lipid biochemical parameters.

Materials and methods. 829 patients completed health and food frequency questionnaires, and underwent anthropometric, arterial stiffness, and blood pressure measurements. Serum concentrations of lipids, glucose, alanine and aspartate aminotransferases, creatinine, uric acid, and C-reactive protein were determined.

Results. The aspartate aminotransferase serum concentration was lower in the breakfast-consuming group $(25.88 \pm 7.05 \mathrm{U} / \mathrm{L})$ compared to non-consumers $(27.75 \pm 10.67 \mathrm{U} / \mathrm{L})$. A lower concentration of creatinine and alanine aminotransferase and a higher concentration of C-reactive protein was found in whole grain product consumers. Individuals consuming more red meat had higher alanine aminotransferase and low-density lipoprotein serum concentrations. Individuals with greater dark chocolate consumption had higher serum concentrations of uric acid ( $5.20 \pm 1.46$ vs. $4.72 \pm 1.18)$ and more intensified arterial stiffness (peak-to-peak time $213.86 \pm 54.98 \mathrm{~ms}$ vs. $238.70 \pm 60.83 \mathrm{~ms})$.

Conclusions. The investigated dietary patterns had a significant impact on serum lipid concentrations, biochemical markers of liver and renal function and inflammation, and arterial stiffness. High consumption of red meat and dark chocolate intensified cardiovascular risk, contrary to the intake of whole grain products.
\end{abstract}

Keywords: dietary patterns, arterial stiffness, glucose, lipid profile, liver function, renal function

Funding: This research received no external funding. 


\section{INTRODUCTION}

According to the Lalonde Report on lifestyle, diet is the lifestyle factor that most significantly affects the health of humans (Lalonde, 1974). It has been shown that the consumption of certain types of foods and meals may have a significant effect on health, especially the consumption of breakfast, which increases the risk of metabolic syndrome when omitted; salty meals, high consumption of which may lead to hypertension; whole grain products, consumption of which decreases all-cause mortality; and dark chocolate. This has caused increasing scientific interest worldwide (Al-Dujaili et al., 2015; Cocate et al., 2015; Gomes et al., 2017; Huang et al., 2015; Meksawan et al., 2014). Skipping breakfast may result in high waist circumference and increased blood glucose and triglyceride levels compared to regular breakfast eating (Meksawan et al., 2014). High red meat consumption ( $\geq 81.5 \mathrm{~g} / \mathrm{d}$ ) correlates with high occurrence of central obesity ( $60 \%)$, hypertriglyceridaemia ( $43 \%)$, and metabolic syndrome (35\%) (Cocate et al., 2015). Whole grain consumers in the highest quintile of consumption were characterised by $6 \%$ lower all-cause mortality compared to those in the lowest quintile (Huang et al., 2015). Dark chocolate consumption with high levels of polyphenols compared to dark chocolate consumption with low levels of polyphenols counteracts the increase in body mass index $(0.17$ vs. $0.32 \mathrm{~kg} /$ $\left.\mathrm{m}^{2}, p=0.007\right)$, blood glucose ( 0.44 vs. $1.08 \mathrm{mmol} / \mathrm{l}$, $p=0.041)$ and triglycerides $(0.13$ vs. $0.23 \mathrm{mmol} / \mathrm{l}$, $p=0.008)$ connected with fat and energy intake in chocolate (Al-Dujaili et al., 2015). Lee et al. (2016) examined the relationship between eating breakfast and blood pressure; body mass index (BMI); serum concentrations of low-density lipoprotein (LDL-C), haemoglobin ( $\mathrm{Hb}$ ), and red blood cells (RBCs); and the occurrence of metabolic disorders. The population that omitted breakfast, defined as subjects not eating any breakfast for a week, had higher blood pressure; higher serum concentrations of LDL-C, $\mathrm{Hb}$, and RBCs; and the rare occurrence of metabolic disorders compared to the population who ate breakfast more than 5 times per week. As for salty meals, the results of a range of studies support the thesis that a diet high in salt increases blood pressure. For example, in normalpressure rats fed a high salt diet, blood pressure was significantly elevated compared to animals on a regular diet after 5 days $(165 \pm 4$ vs. $146 \pm 5 \mathrm{~mm} \mathrm{Hg}, P$ $=0.03$ ) (Gomes et al., 2017; Oh et al., 2015; Stepien et al., 2018; Szulińska et al., 2017; Walkowska et al., 2015). Tighe et al. (2010) found that the consumption of wholegrain products and oatmeal decreased the levels of serum glucose, cholesterol, and C-reactive protein (CRP) after 12 weeks. Strong positive associations between red meat consumption and serum concentrations of alanine aminotransferase (ALT), CRP, high-density lipoprotein (HDL-C), and triglycerides (TGs) were also observed (Montonen et al., 2013). In studies conducted so far, the effects of dark chocolate intake have mainly been considered in reference to glucose and cholesterol serum concentrations and blood pressure. The beneficial effects of cocoa-rich dark chocolate on these biochemical markers have also been confirmed (Mellor et al., 2010; McKeown et al., 2014; Tokede et al., 2011).

Until now, studies on the effects of the consumption of breakfast, salty meals, red meat, whole grain products, and dark chocolate have mainly investigated the influence of these dietary factors on serum glucose concentrations, lipid profiles, whole blood morphological analysis, and blood pressure. So far, the impact of the consumption of these five meals and products on parameters such as serum concentrations of creatinine, uric acid, aspartate aminotransferase (ASP), ALT, CRP, and glomerular filtration rate (GFR) remains poorly understood. To the best of our knowledge, our study is the first to investigate the influence of the consumption of breakfast, salty meals, red meat, whole grain products, and dark chocolate on arterial stiffness, biochemical parameters of glucose and lipid metabolism, liver and renal function, and biochemical markers of inflammation. Moreover, no such study has been conducted in the Greater Poland (Wielkopolska) region so far. Despite a number of preventive programs, Poland remains in a group of countries with high cardiovascular risk (Trzeciak et al., 2017). Thus, studies on factors influencing cardiovascular risk parameters, and dietary patterns in this range, are urgently needed.

The aim of our study was to assess the effects of the consumption of breakfast, salty meals, red meat, whole grain products, and dark chocolate on serum glucose and lipid concentrations, serum biochemical 
parameters of liver and renal function and inflammation, whole blood morphology, blood pressure, and arterial stiffness in a population of the Greater Poland (Wielkopolska) region.

\section{MATERIALS AND METHODS}

\section{Study design}

The study protocol was approved by the Ethics Committee of the Poznan University of Medical Sciences (approval no. 359/15). Informed written consent was obtained from all participants. The study was performed between March 2016 and September 2017 in 7 randomly chosen cities of the Greater Poland (Wielkopolska) region located in western Poland: Ostrów Wielkopolski, Chodzież, Pniewy, Koziegłowy, Kępno, Mosina, and Pleszew. Randomly selected citizens completed a self-reported standardised health questionnaire and a self-reported standardised food frequency questionnaire. All subjects subsequently underwent anthropometric measurements, arterial stiffness measurements, and blood pressure measurements. Blood samples were collected from all participants.

\section{Study patients}

One thousand one hundred and twenty-eight subjects aged 18-70 years were recruited. The inclusion criteria were as follows: written informed consent, $\geq 18$ years of age, ability to complete the health questionnaire and food frequency questionnaire, and citizenship of the Greater Poland (Wielkopolska) region. The exclusion criteria were as follows: $<18$ years of age, inability to complete the health questionnaire and food frequency questionnaire, lack of citizenship in the Greater Poland (Wielkopolska) region, or the presence of a severe health condition (i.e. cancer) requiring implementation of a medically prescribed diet.

After analysis of the health questionnaire and food frequency questionnaire, 299 subjects with missing data were excluded. Eight hundred and twenty-nine (587 women and $242 \mathrm{men}$ ) subjects met all the inclusion criteria, had no exclusion criteria and no missing data, and underwent statistical analysis.

The standardised food frequency questionnaire (FFQ) was used. The FFQ included five questions on the consumption of the following five analysed dietary parameters: red meat, dark chocolate, whole grain products, salty meals, and breakfast. For each question, the frequency of consumption was rated on a six-point scale: 1 - never, 2 - seldom (1-2 times per month), 3 - one time a week, 4 - two times a week, 5 four times a week, and 6 - twice a day or more. The FFQ was self-completed by the patient in conditions ensuring anonymity.

\section{Study groups}

Five analyses were performed separately for each of the following five dietary parameters: consumption of red meat, dark chocolate, whole grain products, salty meals, and breakfast. For each analysis, the patients were divided into two groups: a group who reported eating the foods listed above four times a week or more (answer no. 5 and 6 in FFQ; high consumption group - HC) and a group who reported eating these foods seldomly (1-2 times per month) or not at all (answer no. 1 and 2 in FFQ; low consumption group LC). In each analysis, the investigated groups were homogenous in relation to the other four unanalysed parameters. The baseline characteristics of the patients are shown in Table 1. The number of patients included in each five analyses are presented in Tables 2-6.

Table 1. Characteristic of the study population

\begin{tabular}{lc}
\hline & Mean \pm SD \\
\hline$N$ & 829 \\
Women, \% & 70.81 \\
Men, \% & 29.19 \\
Age, years & $54.49 \pm 14.59$ \\
BMI, kg/m ${ }^{2}$ & $27.59 \pm 7.73$ \\
Hyperlipidaemia, \% & 37.00 \\
Atherosclerosis, \% & 7.50 \\
Hypertension, \% & 32.00 \\
Type-2 diabetes mellitus, \% & 8.20 \\
Ischaemic heart disease, \% & 10.50 \\
Brain stroke, \% & 2.10 \\
Obesity, \% & 27.40 \\
\hline
\end{tabular}




\section{Health questionnaire}

A standardised health questionnaire was used. The questionnaire included questions on date of birth, sex, citizenship, and health conditions such as hyperlipidaemia, hypertension, obesity, ischemic heart disease, type 2 diabetes mellitus, atherosclerosis, and stroke. The health questionnaire was self-completed by the patient in conditions ensuring anonymity.

\section{Anthropometry}

Anthropometric measurements were obtained in patients wearing light clothes, without shoes, who fasted after resting all night. Body mass was measured to the nearest $0.1 \mathrm{~kg}$ with the use of the same electronic weighing scale for all participants (WPT 100/200 OW; Radwag, Radom, Poland), and height was measured to the nearest $1 \mathrm{~cm}$ with the use of a manual stadiometer (WPT 100/200 OW; Radwag, Radom, Poland). The BMI was calculated by dividing the body mass $[\mathrm{kg}]$ by the height $[\mathrm{m}]$ squared.

\section{Blood pressure measurement}

Blood pressure was measured using a digital electronic tensiometer (705IT, Omron, Kyoto, Japan). Large or regular cuffs were used, depending on the subject's arm circumference. Systolic blood pressure (SBP), diastolic blood pressure (DBP), and heart rate (HR) measurements were performed in fasting subjects after a night of rest following 15 minutes of rest in the sitting position, with legs uncrossed and back and arms supported.

\section{Arterial stiffness measurements}

Arterial stiffness measurements were performed using a non-invasive method (photoplethysmography) after a full night of rest. Pulse Trace PCA2 (CareFusion Corporation, San Diego, CA, USA) estimates large artery stiffness from the pulse waveform obtained from a finger by recording digital volume pulse (DVP) (Alaei-Shahmiri et al., 2015). The following three parameters were calculated: peak to peak time (PPT), defined as time from systolic inflection point or first peak to second peak or inflection point; stiffness index (SI), defined as the subject's height divided by PPT; and reflection index (RI), defined as the height of the second peak or inflection point divided by the height of the first peak.

\section{Blood sample collection}

Blood samples were collected in the morning after a night of rest and a 10 -hour fast and after $30 \mathrm{~min}$ of rest in the supine position. Blood was collected from an ulnar vein into serum-separated tubes and in another tube with ethylenediaminetetraacetic acid (EDTA) to obtain whole blood. Serum samples were frozen immediately after preparation and stored at $-80^{\circ} \mathrm{C}$ until analysis.

\section{Biochemical measurements}

Whole blood morphological parameters and the serum concentration of total cholesterol (TCh), HDL-C, TGs, and glucose $(\mathrm{G})$ were assayed using routine enzymatic methods in a clinical diagnostic laboratory (Aglab Laboratory, Poznan, Poland). The concentration of LDL-C in the serum was calculated using the Friedewald formula (Friedewald et al., 1972). The serum concentrations of ALT, ASP, creatinine (Creat), and uric acid (UA) were estimated using the colorimetric method. The serum concentration of CRP was measured using a rapid-spot immunoprecipitate assay method. The glomerular filtration rate (GFR) was calculated with the Cockcroft-Gault formula (National Kidney Foundation, 2002).

\section{Statistical analysis}

Statistical analysis was performed with Statistica for Windows 10.0 (StatSoft, Krakow, Poland). The results are shown as arithmetic means \pm standard deviations (SDs). Normal distribution of the data was determined using the Shapiro-Wilk test. Comparison between groups was performed using the Mann-Whitney test and the $\mathrm{chi}^{2}$ test. Regression analysis was used to assess the relationship between variables. A $p$-value of less than 0.05 was regarded as significant. It was calculated that a sample size of at least 45 subjects in an analyzed group would yield at least $80 \%$ power of detecting an intervention effect that was statistically significant at the $0.05 \alpha$ level.

\section{RESULTS}

The study population consisted of 829 subjects (587 women and $242 \mathrm{men}$ ). The characteristics of the subjects, including the percentage of participants with metabolic disorders reported in the health questionnaire, are presented in Table 1. 
Table 2 presents a comparison of the analysed parameters between two study groups according to breakfast consumption. The RBC concentration was higher and the ASP serum concentration was lower in the HC group than in the LC group. It was observed that in the group that skipped breakfast subjects

Table 2. Comparison of analysed parameters between two study groups according to breakfast consumption

\begin{tabular}{|c|c|c|c|}
\hline $\begin{array}{c}\text { Parameters } \\
n=215\end{array}$ & $\begin{array}{l}\text { High consumption } \\
\text { group - group } 1 \\
\text { mean } \pm \text { SD } \\
n=120\end{array}$ & $\begin{array}{c}\text { Low consumption } \\
\text { group - group } 2 \\
\text { mean } \pm \text { SD } \\
n=95\end{array}$ & $p$ \\
\hline 1 & 2 & 3 & 4 \\
\hline \multicolumn{4}{|c|}{ Characteristic of the groups } \\
\hline Women, \% & 48 & 68 & \\
\hline Men, $\%$ & 52 & 32 & \\
\hline Age & $54.34 \pm 14.92$ & $54.26 \pm 14.77$ & NS \\
\hline BMI & $26.84 \pm 4.82$ & $27.41 \pm 5.12$ & NS \\
\hline Hyperlipidaemia, \% & 32.00 & 29.70 & NS \\
\hline Atherosclerosis, $\%$ & 6.20 & 7.70 & NS \\
\hline Hypertension, \% & 34.30 & 36.80 & NS \\
\hline Type-2 diabetes mellitus, \% & 6.70 & 5.20 & NS \\
\hline Ischaemic heart disease, $\%$ & 7.80 & 9.70 & NS \\
\hline Brain stroke, $\%$ & 2.20 & 3.90 & NS \\
\hline Obesity, \% & 25.70 & 26.50 & NS \\
\hline \multicolumn{4}{|c|}{ Blood pressure and arterial stiffness } \\
\hline $\mathrm{SI}, \mathrm{m} / \mathrm{s}$ & $7.59 \pm 2.77$ & $8.34 \pm 7.33$ & NS \\
\hline PPT, ms & $236.12 \pm 58.58$ & $233.18 \pm 66.98$ & NS \\
\hline RI, \% & $54.74 \pm 16.82$ & $57.52 \pm 25.38$ & NS \\
\hline SBP, mm Hg & $130.76 \pm 25.70$ & $129.36 \pm 25.98$ & NS \\
\hline DBP, mm Hg & $79.62 \pm 11.50$ & $79.43 \pm 10.80$ & NS \\
\hline HR & $75.34 \pm 12.36$ & $77.26 \pm 12.16$ & NS \\
\hline \multicolumn{4}{|c|}{ Haematological parameters } \\
\hline $\mathrm{WBC}, \mathrm{K} / \mu \mathrm{l}$ & $6.81 \pm 1.71$ & $7.15 \pm 1.86$ & NS \\
\hline $\mathrm{RBC}, \mathrm{M} / \mu \mathrm{l}$ & $4.63 \pm 0.42$ & $4.55 \pm 0.41$ & 0.05 \\
\hline $\mathrm{Hb}, \mathrm{g} / \mathrm{Dl}$ & $13.86 \pm 1.24$ & $13.68 \pm 1.14$ & NS \\
\hline HCT, \% & $41.71 \pm 3.87$ & $41.04 \pm 3.43$ & NS \\
\hline $\mathrm{MCV}, \mathrm{fL}$ & $89.88 \pm 4.45$ & $90.37 \pm 4.52$ & NS \\
\hline
\end{tabular}


Table 2 cont.

\begin{tabular}{|c|c|c|c|}
\hline 1 & 2 & 3 & 4 \\
\hline \multicolumn{4}{|c|}{ Biochemical parameters } \\
\hline Creat, mg/dL & $0.78 \pm 0.16$ & $0.76 \pm 0.16$ & NS \\
\hline $\mathrm{eGFR}, \mathrm{ml} / \mathrm{min} / \mathrm{m}^{2}$ & $60.49 \pm 2.72$ & $60.78 \pm 1.38$ & NS \\
\hline $\mathrm{UA}, \mathrm{mg} / \mathrm{Dl}$ & $5.21 \pm 1.35$ & $5.076 \pm 1.24$ & NS \\
\hline $\mathrm{CRP}, \mathrm{mg} / \mathrm{L}$ & $4.34 \pm 6.07$ & $5.30 \pm 7.36$ & NS \\
\hline ASP, U/L & $25.88 \pm 7.05$ & $27.75 \pm 10.67$ & 0.05 \\
\hline ALT, U/L & $27.33 \pm 10.52$ & $27.39 \pm 11.41$ & NS \\
\hline $\mathrm{G}, \mathrm{mg} / \mathrm{dl}$ & $92.69 \pm 19.11$ & $90.34 \pm 15.01$ & NS \\
\hline $\mathrm{TCh}, \mathrm{mg} / \mathrm{Dl}$ & $202.29 \pm 39.18$ & $200.64 \pm 42.78$ & NS \\
\hline HDL-C, mg/Dl & $64.98 \pm 16.11$ & $65.23 \pm 16.40$ & NS \\
\hline LDL-C, mg/Dl & $112.12 \pm 44.43$ & $110.11 \pm 47.82$ & NS \\
\hline $\mathrm{TG}, \mathrm{mg} / \mathrm{Dl}$ & $146.16 \pm 83.70$ & $140.72 \pm 71.34$ & NS \\
\hline
\end{tabular}

$\mathrm{Si}$ - stiffness index, PPT - peak to peak time, Ri - reflection index, SBP - systolic blood pressure, DBP - diastolic blood pressure, HR - heart rate, WBC - white blood cells, RBC red blood cells, $\mathrm{Hb}$ - haemoglobin, $\mathrm{HCT}$ - haematocrit, MCV - mean corpuscular volume, $\mathrm{MCH}$ - mean corpuscular haemoglobin, $\mathrm{MCHC}$ - mean corpuscular haemoglobin concentration, RDW - red cell distribution width, PCT - plateletcrit, PDW - platelet distribution width, MPV - mean platelet volume, Creat - creatinine, eGFR - estimated glomerular filtration rate, UA - uric acid, CRP - C-reactive protein, ASP - aspartate aminotransferase, ALT - alanine aminotransferase, $\mathrm{G}$ - glucose, TCh - total cholesterol, HDL-C - high-density lipoprotein, LDL-C - low-density lipoprotein, TG - triglycerides.

presented a non-significant trend to suffer more often from atherosclerosis, hypertension, ischemic heart disease, and obesity.

Table 3 shows a comparison of the analysed parameters of two study groups according to salty meal consumption. It was found that subjects who more often ate salty meals had lower levels of mean corpuscular volume (MCV) and presented a non-significant trend to suffer more often from type-2 diabetes mellitus, ischemic heart disease, or obesity compared to the LC group.

Table 4 presents a comparison of the analysed parameters of two study groups according to the consumption of wholegrain products. Lower serum concentrations of $\mathrm{Hb}$, Create, and ALT and higher concentrations of CRP were found in the HC group compared to the LC group. Higher frequencies (though without statistical significance) of hyperlipidaemia, atherosclerosis, hypertension, and type-2 diabetes mellitus were observed in the LC group.

Table 5 shows a comparison of the analysed parameters of two study groups according to red meat consumption. Individuals consuming more red meat had significantly higher ALT and LDL-C serum concentrations and significantly lower MCV compared to the LC group. Individuals who ate red meat also (though without statistical significance) had type-2 diabetes mellitus more often than those in the LC group.

Table 6 presents a comparison of the analysed parameters of two study groups according to dark chocolate consumption. Individuals with greater dark chocolate consumption had higher serum concentrations of UA and lower values of PPT than the LC group. The HC group was characterised by a non-significant trend to show a lower occurrence of obesity and atherosclerosis. 
Table 3. Comparison of the analysed parameters of two study groups according to salty meal consumption

\begin{tabular}{|c|c|c|c|}
\hline $\begin{array}{c}\text { Parameters } \\
n=218\end{array}$ & $\begin{array}{l}\text { High consumption } \\
\text { group - group } 3 \\
\text { mean } \pm \text { SD } \\
n=103\end{array}$ & $\begin{array}{c}\text { Low consumption } \\
\text { group - group } 4 \\
\text { mean } \pm \text { SD } \\
n=115\end{array}$ & $p$ \\
\hline 1 & 2 & 3 & 4 \\
\hline \multicolumn{4}{|c|}{ Characteristic of the groups } \\
\hline Women, \% & 50 & 64 & \\
\hline Men, $\%$ & 50 & 36 & \\
\hline Age & $54.07 \pm 14.68$ & $54.77 \pm 14.73$ & NS \\
\hline BMI & $27.49 \pm 5.37$ & $27.71 \pm 9.52$ & NS \\
\hline Hyperlipidaemia, \% & 34.70 & 35.10 & NS \\
\hline Atherosclerosis, $\%$ & 5.70 & 8.50 & NS \\
\hline Hypertension, \% & 33.20 & 36.30 & NS \\
\hline Diabetes mellitus II, \% & 8.40 & 8.30 & NS \\
\hline Ischaemic heart disease, $\%$ & 10.00 & 9.70 & NS \\
\hline Brain stroke, $\%$ & 2.20 & 3.60 & NS \\
\hline Obesity, \% & 27.40 & 26.10 & NS \\
\hline \multicolumn{4}{|c|}{ Blood pressure and arterial stiffness } \\
\hline $\mathrm{SI}, \mathrm{m} / \mathrm{s}$ & $8.44 \pm 8.57$ & $7.95 \pm 5.07$ & NS \\
\hline PPT, ms & $236.31 \pm 66.79$ & $236.53 \pm 68.54$ & NS \\
\hline RI, \% & $53.72 \pm 22.09$ & $54.09 \pm 38.23$ & NS \\
\hline $\mathrm{SBP}, \mathrm{mm} \mathrm{Hg}$ & $133.07 \pm 23.28$ & $131.74 \pm 24.71$ & NS \\
\hline DBP, mm Hg & $81.26 \pm 11.50$ & $80.50 \pm 11.30$ & NS \\
\hline HR & $76.78 \pm 12.84$ & $76.93 \pm 12.50$ & NS \\
\hline \multicolumn{4}{|c|}{ Haematological parameters } \\
\hline $\mathrm{WBC}, \mathrm{K} / \mu \mathrm{l}$ & $7.05 \pm 1.72$ & $6.96 \pm 1.73$ & NS \\
\hline $\mathrm{RBC}, \mathrm{M} / \mu \mathrm{l}$ & $4.59 \pm 0.42$ & $4.56 \pm 0.43$ & NS \\
\hline $\mathrm{Hb}, \mathrm{g} / \mathrm{Dl}$ & $13.75 \pm 1.19$ & $13.76 \pm 1.42$ & NS \\
\hline HCT, \% & $41.29 \pm 3.62$ & $41.35 \pm 3.91$ & NS \\
\hline MCV, fL & $89.77 \pm 6.32$ & $90.63 \pm 4.21$ & 0.02 \\
\hline \multicolumn{4}{|c|}{ Biochemical parameters } \\
\hline Creat, mg/Dl & $0.77 \pm 0.17$ & $0.77 \pm 0.16$ & NS \\
\hline $\mathrm{eGFR}, \mathrm{ml} / \mathrm{min} / \mathrm{m}^{2}$ & $60.73 \pm 1.88$ & $60.46 \pm 3.21$ & NS \\
\hline $\mathrm{UA}, \mathrm{mg} / \mathrm{dL}$ & $5.11 \pm 1.32$ & $5.09 \pm 2.66$ & NS \\
\hline
\end{tabular}


Table 3 cont.

\begin{tabular}{lccl}
\hline & 1 & 3 & 4 \\
\hline CRP, mg/L & $4.25 \pm 3.20$ & $4.04 \pm 4.24$ & NS \\
ASP, U/L & $28.50 \pm 10.76$ & $27.44 \pm 8.93$ & NS \\
ALT, U/L & $30.35 \pm 16.60$ & $29.33 \pm 12.95$ & NS \\
G, mg/dl & $91.45 \pm 16.46$ & $91.89 \pm 18.28$ & NS \\
TCh, mg/dL & $199.23 \pm 40.65$ & $201.34 \pm 43.15$ & NS \\
HDL-C, mg/dL & $65.52 \pm 16.90$ & $64.93 \pm 17.60$ & NS \\
LDL-C, mg/dL & $107.98 \pm 39.80$ & $109.55 \pm 42.64$ & NS \\
TG, mg/dL & $138.01 \pm 76.21$ & $137.64 \pm 77.60$ & NS \\
\hline
\end{tabular}

$\mathrm{Si}$ - stiffness index, PPT - peak to peak time, Ri - reflection index, SBP - systolic blood pressure, DBP - diastolic blood pressure, HR - heart rate, WBC - white blood cells, RBC red blood cells, $\mathrm{Hb}$ - haemoglobin, HCT - haematocrit, MCV - mean corpuscular volume, $\mathrm{MCH}$ - mean corpuscular haemoglobin, $\mathrm{MCHC}$ - mean corpuscular haemoglobin concentration, RDW - red cell distribution width, PCT - plateletcrit, PDW - platelet distribution width, MPV - mean platelet volume, Creat - creatinine, eGFR - estimated glomerular filtration rate, UA - uric acid, CRP - C-reactive protein, ASP - aspartate aminotransferase, ALT - alanine aminotransferase, G - glucose, TCh - total cholesterol, HDL-C - high-density lipoprotein, LDL-C - low-density lipoprotein, TG - triglycerides.

Table 4. Comparison of the analysed parameters of two study groups according to the consumption of wholegrain products

\begin{tabular}{lccc}
\hline \multicolumn{1}{c}{$\begin{array}{c}\text { Parameters } \\
n=213\end{array}$} & $\begin{array}{c}\text { High consumption } \\
\text { group }- \text { group } \\
\text { mean } \pm \text { SD } \\
n=122\end{array}$ & $\begin{array}{c}\text { Low consumption } \\
\text { group }- \text { group } 6 \\
\text { mean } \pm \text { SD } \\
n=91\end{array}$ & $p$ \\
\hline Characteristic of the groups & 3 & 4 \\
\hline Women, \% & 43 & 72 & \\
Men, \% & 57 & 28 & NS \\
Age & $54.16 \pm 14.89$ & $55.30 \pm 14.01$ & NS \\
BMI & $27.97 \pm 6.03$ & $27.25 \pm 5.44$ & NS \\
Hyperlipidaemia, \% & 33.10 & 36.10 & NS \\
Atherosclerosis, \% & 6.30 & 8.20 & NS \\
Hypertension, \% & 31.90 & 36.10 & NS \\
Diabetes mellitus II, \% & 8.10 & 8.20 & NS \\
Ischaemic heart disease, \% & 9.40 & 7.40 & NS \\
Brain stroke, \% & 8.80 & 6.60 & NS \\
Obesity, \% & 31.30 & 26.20 & \\
\hline
\end{tabular}


Skrypnik, K., Bogdanski, P., Kubasik, M., Wawrzyniak, N., Markuszewski, L., Suliburska, J. (2020). The influence of dietary patterns on arterial stiffness, lipid metabolism, and liver and renal function in the population of Greater Poland. Acta Sci. Pol. Technol. Aliment., 19(3), 301-318. http://dx.doi.org/10.17306/J.AFS.2020.0848

Table 4 cont.

\begin{tabular}{|c|c|c|c|}
\hline 1 & 2 & 3 & 4 \\
\hline \multicolumn{4}{|c|}{ Blood pressure and arterial stiffness } \\
\hline $\mathrm{SI}, \mathrm{m} / \mathrm{s}$ & $7.59 \pm 2.18$ & $7.84 \pm 3.64$ & NS \\
\hline PPT, ms & $235.51 \pm 58.04$ & $234.06 \pm 56.58$ & NS \\
\hline RI, \% & $51.35 \pm 16.31$ & $51.36 \pm 17.77$ & NS \\
\hline SBP, mm Hg & $131.98 \pm 24.13$ & $133.89 \pm 18.95$ & NS \\
\hline $\mathrm{DBP}, \mathrm{mm} \mathrm{Hg}$ & $79.77 \pm 9.94$ & $80.30 \pm 11.53$ & NS \\
\hline HR & $77.98 \pm 12.15$ & $75.75 \pm 10.92$ & NS \\
\hline \multicolumn{4}{|c|}{ Haematological parameters } \\
\hline $\mathrm{WBC}, \mathrm{K} / \mu 1$ & $6.95 \pm 1.54$ & $7.19 \pm 1.82$ & NS \\
\hline $\mathrm{RBC}, \mathrm{M} / \mu \mathrm{l}$ & $4.57 \pm 0.43$ & $4.62 \pm 0.41$ & NS \\
\hline $\mathrm{Hb}, \mathrm{g} / \mathrm{Dl}$ & $13.55 \pm 1.55$ & $13.90 \pm 1.17$ & 0.04 \\
\hline НCТ, \% & $41.09 \pm 3.66$ & $41.712 \pm 3.69$ & NS \\
\hline $\mathrm{MCV}, \mathrm{fL}$ & $90.03 \pm 5.11$ & $90.62 \pm 3.72$ & NS \\
\hline \multicolumn{4}{|c|}{ Biochemical parameters } \\
\hline Creat, $\mathrm{mg} / \mathrm{dL}$ & $0.74 \pm 0.18$ & $0.78 \pm 0.16$ & 0.05 \\
\hline $\mathrm{eGFR}, \mathrm{ml} / \mathrm{min} / \mathrm{m}^{2}$ & $60.66 \pm 1.81$ & $60.54 \pm 2.93$ & NS \\
\hline $\mathrm{UA}, \mathrm{mg} / \mathrm{Dl}$ & $4.90 \pm 1.32$ & $5.06 \pm 1.20$ & NS \\
\hline $\mathrm{CRP}, \mathrm{mg} / \mathrm{L}$ & $4.19 \pm 2.58$ & $3.77 \pm 1.49$ & 0.05 \\
\hline ASP, U/L & $26.71 \pm 7.73$ & $28.08 \pm 9.93$ & NS \\
\hline ALT, U/L & $26.93 \pm 10.05$ & $29.50 \pm 13.95$ & 0.04 \\
\hline $\mathrm{G}, \mathrm{mg} / \mathrm{dl}$ & $92.62 \pm 17.81$ & $93.29 \pm 20.07$ & NS \\
\hline TCh, mg/Dl & $201.67 \pm 42.02$ & $204.04 \pm 36.40$ & NS \\
\hline HDL-C, mg/Dl & $65.08 \pm 18.32$ & $65.80 \pm 16.46$ & NS \\
\hline LDL-C, mg/Dl & $106.99 \pm 36.27$ & $112.20 \pm 39.02$ & NS \\
\hline $\mathrm{TG}, \mathrm{mg} / \mathrm{Dl}$ & $142.24 \pm 77.71$ & $143.68 \pm 71.72$ & NS \\
\hline
\end{tabular}

$\mathrm{Si}$ - stiffness index, PPT - peak to peak time, Ri - reflection index, SBP - systolic blood pressure, DBP - diastolic blood pressure, HR - heart rate, WBC - white blood cells, RBC red blood cells, $\mathrm{Hb}$ - haemoglobin, HCT - haematocrit, MCV - mean corpuscular volume, $\mathrm{MCH}$ - mean corpuscular haemoglobin, $\mathrm{MCHC}$ - mean corpuscular haemoglobin concentration, RDW - red cell distribution width, PCT - plateletcrit, PDW - platelet distribution width, MPV - mean platelet volume, Creat - creatinine, eGFR - estimated glomerular filtration rate, $\mathrm{UA}$ - uric acid, CRP - C-reactive protein, ASP - aspartate aminotransferase, ALT - alanine aminotransferase, G - glucose, TCh - total cholesterol, HDL-C - high-density lipoprotein, LDL-C - low-density lipoprotein, TG - triglycerides. 
Table 5. Comparison of the analysed parameters of two study groups according to red meat consumption

\begin{tabular}{|c|c|c|c|}
\hline $\begin{array}{c}\text { Parameters } \\
n=221\end{array}$ & $\begin{array}{c}\text { High consumption } \\
\text { group - group } 7 \\
\text { mean } \pm \text { SD } \\
n=50\end{array}$ & $\begin{array}{c}\text { Low consumption } \\
\text { group - group } 8 \\
\text { mean } \pm \text { SD } \\
n=171\end{array}$ & $p$ \\
\hline 1 & 2 & 3 & 4 \\
\hline \multicolumn{4}{|c|}{ Characteristic of the groups } \\
\hline Women, \% & 58 & 57 & \\
\hline Men, $\%$ & 42 & 43 & \\
\hline Age & $53.26 \pm 14.88$ & $55.60 \pm 13.69$ & NS \\
\hline BMI & $27.11 \pm 4.61$ & $27.27 \pm 5.44$ & NS \\
\hline Hyperlipidaemia, \% & 32.00 & 37.20 & NS \\
\hline Atherosclerosis, $\%$ & 4.00 & 8.10 & NS \\
\hline Hypertension, $\%$ & 24.00 & 33.70 & NS \\
\hline Type- 2 diabetes mellitus, $\%$ & 10.00 & 7.00 & NS \\
\hline Ischaemic heart disease, $\%$ & 10.00 & 11.60 & NS \\
\hline Brain stroke, $\%$ & 2.00 & 2.30 & NS \\
\hline Obesity, \% & 18.00 & 27.90 & NS \\
\hline \multicolumn{4}{|c|}{ Blood pressure and arterial stiffness } \\
\hline $\mathrm{SI}, \mathrm{m} / \mathrm{s}$ & $7.13 \pm 1.86$ & $8.24 \pm 9.03$ & NS \\
\hline $\mathrm{PPT}, \mathrm{ms}$ & $244.70 \pm 52.02$ & $236.93 \pm 61.38$ & NS \\
\hline RI, \% & $50.85 \pm 16.65$ & $50.86 \pm 16.29$ & NS \\
\hline SBP, mm Hg & $129.90 \pm 15.52$ & $133.51 \pm 22.29$ & NS \\
\hline DBP, $\mathrm{mm} \mathrm{Hg}$ & $79.18 \pm 11.37$ & $80.33 \pm 10.91$ & NS \\
\hline HR & $78.87 \pm 12.95$ & $77.19 \pm 11.43$ & NS \\
\hline \multicolumn{4}{|c|}{ Haematological parameters } \\
\hline $\mathrm{WBC}, \mathrm{K} / \mu \mathrm{l}$ & $7.16 \pm 1.64$ & $7.03 \pm 1.70$ & NS \\
\hline $\mathrm{RBC}, \mathrm{M} / \mu \mathrm{l}$ & $4.61 \pm 0.39$ & $4.56 \pm 0.42$ & NS \\
\hline $\mathrm{Hb}, \mathrm{g} / \mathrm{Dl}$ & $13.62 \pm 1.04$ & $13.68 \pm 1.08$ & NS \\
\hline НCT, \% & $41.00 \pm 3.21$ & $41.15 \pm 3.55$ & NS \\
\hline $\mathrm{MCV}, \mathrm{fL}$ & $89.10 \pm 3.49$ & $90.60 \pm 4.59$ & 0.04 \\
\hline \multicolumn{4}{|c|}{ Biochemical parameters } \\
\hline Creat, mg/Dl & $0.77 \pm 0.14$ & $0.74 \pm 0.16$ & NS \\
\hline $\mathrm{eGFR}, \mathrm{ml} / \mathrm{min} / \mathrm{m}^{2}$ & $60.80 \pm 1.06$ & $60.76 \pm 1.92$ & NS \\
\hline $\mathrm{UA}, \mathrm{mg} / \mathrm{Dl}$ & $4.75 \pm 1.33$ & $4.82 \pm 1.23$ & NS \\
\hline
\end{tabular}


Table 5 cont.

\begin{tabular}{lccc}
\hline & 1 & 3 & 4 \\
\hline CRP, mg/L & $3.58 \pm 1.16$ & $4.18 \pm 2.45$ & NS \\
ASP, U/L & $28.04 \pm 7.70$ & $28.10 \pm 11.81$ & NS \\
ALT, U/L & $30.51 \pm 13.32$ & $25.95 \pm 9.18$ & 0.01 \\
G, mg/dl & $90.64 \pm 16.17$ & $93.20 \pm 17.22$ & NS \\
TCh, mg/Dl & $204.89 \pm 31.99$ & $199.98 \pm 41.84$ & NS \\
HDL-C, mg/Dl & $65.31 \pm 15.63$ & $66.34 \pm 16.54$ & NS \\
LDL-C, mg/Dl & $117.84 \pm 44.80$ & $105.97 \pm 35.97$ & 0.05 \\
TG, mg/Dl & $140.70 \pm 85.60$ & $138.26 \pm 79.24$ & NS \\
\hline
\end{tabular}

$\mathrm{Si}$ - stiffness index, PPT - peak to peak time, $\mathrm{Ri}$ - reflection index, SBP - systolic blood pressure, DBP - diastolic blood pressure, HR - heart rate, WBC - white blood cells, RBC red blood cells, $\mathrm{Hb}$ - haemoglobin, $\mathrm{HCT}$ - haematocrit, MCV - mean corpuscular volume, $\mathrm{MCH}$ - mean corpuscular haemoglobin, MCHC - mean corpuscular haemoglobin concentration, RDW - red cell distribution width, PCT - plateletcrit, PDW - platelet distribution width, MPV - mean platelet volume, Creat - creatinine, eGFR - estimated glomerular filtration rate, UA - uric acid, CRP - C-reactive protein, ASP - aspartate aminotransferase, ALT - alanine aminotransferase, G - glucose, TCh - total cholesterol, HDL-C - high-density lipoprotein, LDL-C - low-density lipoprotein, TG - triglycerides.

Table 6. Comparison of the analysed parameters of two study groups according to dark chocolate consumption

\begin{tabular}{lccc}
\hline \multicolumn{1}{c}{$\begin{array}{c}\text { Parameters } \\
n=217\end{array}$} & $\begin{array}{c}\text { High consumption } \\
\text { group }- \text { group } \\
\text { mean } \pm \text { SD } \\
n=62\end{array}$ & $\begin{array}{c}\text { Low consumption } \\
\text { group }- \text { group } 10 \\
\text { mean } \pm \text { SD } \\
n=155\end{array}$ & $p<0.05$ \\
\hline 1 & 2 & 3 & 4 \\
\hline Characteristics of the groups & & \\
\hline Women, \% & 39 & 64 & NS \\
Agen, $\%$ & 61 & 36 & NS \\
BMI & $55.91 \pm 14.52$ & $55.26 \pm 14.00$ & NS \\
Hyperlipidaemia, \% & $26.98 \pm 4.68$ & $27.09 \pm 5.46$ & NS \\
Atherosclerosis, \% & 42.30 & 37.00 & NS \\
Hypertension, \% & 4.00 & 8.10 & NS \\
Type-2 diabetes mellitus, \% & 36.40 & 33.50 & NS \\
Ischaemic heart disease, \% & 9.00 & 6.90 & NS \\
Brain stroke, \% & 16.70 & 11.60 & NS \\
Obesity, \% & 4.50 & 2.30 & \\
\hline
\end{tabular}


Table 6 cont.

\begin{tabular}{|c|c|c|c|}
\hline 1 & 2 & 3 & 4 \\
\hline \multicolumn{4}{|c|}{ Blood pressure and arterial stiffness } \\
\hline $\mathrm{SI}, \mathrm{m} / \mathrm{s}$ & $8.08 \pm 2.34$ & $8.23 \pm 9.47$ & NS \\
\hline PPT, ms & $213.86 \pm 54.98$ & $238.70 \pm 60.83$ & 0.02 \\
\hline RI, \% & $55.12 \pm 14.36$ & $50.36 \pm 15.37$ & NS \\
\hline $\mathrm{SBP}, \mathrm{mm} \mathrm{Hg}$ & $135.03 \pm 27.09$ & $132.77 \pm 22.64$ & NS \\
\hline $\mathrm{DBP}, \mathrm{mm} \mathrm{Hg}$ & $80.75 \pm 12.58$ & $80.18 \pm 10.97$ & NS \\
\hline HR & $75.03 \pm 10.92$ & $75.74 \pm 9.95$ & NS \\
\hline \multicolumn{4}{|c|}{ Haematological parameters } \\
\hline $\mathrm{WBC}, \mathrm{K} / \mu \mathrm{l}$ & $6.91 \pm 1.74$ & $7.01 \pm 1.67$ & NS \\
\hline $\mathrm{RBC}, \mathrm{M} / \mu 1$ & $4.53 \pm 0.39$ & $4.52 \pm 0.39$ & NS \\
\hline $\mathrm{Hb}, \mathrm{g} / \mathrm{D} 1$ & $13.41 \pm 1.76$ & $13.52 \pm 0.96$ & NS \\
\hline НCT, \% & $40.86 \pm 2.97$ & $40.65 \pm 3.18$ & NS \\
\hline $\mathrm{MCV}, \mathrm{fL}$ & $90.40 \pm 4.84$ & $90.36 \pm 4.57$ & NS \\
\hline \multicolumn{4}{|c|}{ Biochemical parameters } \\
\hline Creat, mg/Dl & $0.75 \pm 0.16$ & $0.73 \pm 0.16$ & NS \\
\hline $\mathrm{eGFR}, \mathrm{ml} / \mathrm{min} / \mathrm{m}^{2}$ & $61.00 \pm 0.00$ & $60.74 \pm 2.01$ & NS \\
\hline $\mathrm{UA}, \mathrm{mg} / \mathrm{Dl}$ & $5.20 \pm 1.46$ & $4.72 \pm 1.18$ & 0.01 \\
\hline $\mathrm{CRP}, \mathrm{mg} / \mathrm{L}$ & $3.91 \pm 2.12$ & $4.22 \pm 2.46$ & NS \\
\hline ASP, U/L & $28.93 \pm 11.14$ & $27.60 \pm 11.22$ & NS \\
\hline ALT, U/L & $28.02 \pm 12.55$ & $27.06 \pm 12.26$ & NS \\
\hline $\mathrm{G}, \mathrm{mg} / \mathrm{dl}$ & $90.50 \pm 13.69$ & $93.70 \pm 18.66$ & NS \\
\hline $\mathrm{TCh}, \mathrm{mg} / \mathrm{Dl}$ & $203.63 \pm 38.91$ & $200.50 \pm 42.38$ & NS \\
\hline HDL-C, mg/Dl & $66.63 \pm 17.21$ & $67.27 \pm 16.79$ & NS \\
\hline LDL-C, mg/Dl & $107.72 \pm 34.38$ & $105.93 \pm 10.06$ & NS \\
\hline $\mathrm{TG}, \mathrm{mg} / \mathrm{Dl}$ & $136.13 \pm 63.77$ & $139.15 \pm 83.66$ & NS \\
\hline
\end{tabular}

$\mathrm{Si}$ - stiffness index, PPT - peak to peak time, Ri - reflection index, SBP - systolic blood pressure, DBP - diastolic blood pressure, HR - heart rate, WBC - white blood cells, RBC red blood cells, $\mathrm{Hb}$ - haemoglobin, $\mathrm{HCT}$ - haematocrit, $\mathrm{MCV}$ - mean corpuscular volume, $\mathrm{MCH}$ - mean corpuscular haemoglobin, $\mathrm{MCHC}$ - mean corpuscular haemoglobin concentration, RDW - red cell distribution width, PCT - plateletcrit, PDW - platelet distribution width, MPV - mean platelet volume, Creat - creatinine, eGFR - estimated glomerular filtration rate, $\mathrm{UA}$ - uric acid, CRP - C-reactive protein, ASP - aspartate aminotransferase, ALT - alanine aminotransferase, $\mathrm{G}$ - glucose, TCh - total cholesterol, HDL-C - high-density lipoprotein, LDL-C - low-density lipoprotein, TG - triglycerides. 
The regression analysis showed that red meat consumption $(\beta=0.17, p=0.041)$ and BMI $(\beta=0.434$, $p=0.0003)$ are independent predictors of serum ALT concentration (among age and other analysed dietary patterns). It was also found that dark chocolate consumption $(\beta=0.16, p=0.031)$ and BMI $(\beta=411$, $p=0.0002)$ had a significant effect on the serum UA concentration (among age and other analysed dietary patterns).

\section{DISCUSSION}

Our study revealed that dark chocolate consumption has a significant influence on arterial stiffness. Dark chocolate consumers had lower PPT compared to nonconsumers, which indicates higher large artery stiffness in the group of subjects consuming dark chocolate (Millasseau et al., 2006). The study showed that two seemingly healthy food products can also have a negative effect on health. Dark chocolate consumption has been found to be an independent prognostic factor for increases in uric acid serum concentrations. In addition, the intake of whole grains has been shown to increase CRP levels in the blood.

Our observations regarding the percentage of people suffering from hypertension, diabetes, or obesity are consistent with the incidence of these diseases in Poland and Europe.

Our results show that skipping breakfast may decrease RBC levels and increase ASP concentrations. Such results imply that skipping breakfast may lead to anemia and disordered liver function. Other studies have indicated that populations who skip breakfast have a worse quality diet and irregular meal intake (Kant et al., 2008; Min et al., 2011). This decreased quality of diet is associated with a lower intake of nutrients, including iron $(\mathrm{Fe})$ and vitamin $\mathrm{C}$, which simultaneously affects $\mathrm{Fe}$ absorption (Atanassova and Tzatchev, n.d.). Iron transport by transferrin is essential for the synthesis of haemoglobin and for RBCs, and its lower levels may result in decreased concentrations of RBCs in the blood (Johnson-Wimbley and Graham, 2011). The omission of breakfast, a lower quality diet, and the resulting lower nutrient intake may have negative effects on liver function, which may, in turn, increase ASP concentrations. It is known that higher ASP levels are associated with obesity and heart disease (Giboney, 2005), and in this study we also observed that these conditions - along with hypertension and atherosclerosis - occur more often in those who skip breakfast. A similar correlation was observed in a cohort study of the American population where skipping breakfast was associated with an increased number of deaths due to cardiovascular disease.

In contrast to our findings, Lee et al. (2016) showed that skipping breakfast increases RBC concentrations. Another author showed that populations that consume breakfast more often have higher concentrations of iron in their serum (Min et al., 2011). Furthermore, the omission of breakfast leads to greater consumption of meat at dinner, which may explain the higher iron status (Lee et al., 2016). Other studies have demonstrated that omitting breakfast may have a negative impact on TCh, G, HDL-C, LDL-C, and TG concentrations, suggesting a greater risk of hypercholesterolemia and metabolic syndrome, hypertension, and type- 2 diabetes mellitus. Previous studies have indicated the positive impact of not eating breaking on triglycerides (Min et al., 2011), showing that populations who skipped breakfast had lower TG levels. The reason for the divergent results may be the design of the research, such as different breakfast compositions, energy balances, age groups, fasting periods before eating breakfast, or forgetfulness regarding consumption.

Our results also showed that subjects who ate salty meals more often had lower levels of MCV than subjects who ate salty meals less often. The reason for the lower MCV is not clear, but we also observed low levels of MCV in subjects that consumed more red meat. Some authors have suggested that these two eating behaviours are related. Populations who consume red meat more often also consume more salt, as well as more processed red meat. These authors also indicated a greater prevalence of type- 2 diabetes mellitus in both the red meat and salt consumption groups, which is similar to our findings. The results from our present study also indicate a likely association between the occurrence of type-2 diabetes mellitus, ischemic heart disease, and obesity and more frequent salty meals. Salty meal eaters also had higher blood pressure than non-eaters, which has also been observed by other authors. In contrast to our findings, other authors have found associations between salty meals and red 
meat consumption and a high risk of developing hypertension (Gomes et al., 2017; Larsson et al., 2011; Oh et al., 2015; Walkowska et al., 2015). Interestingly, in a study by Wahab, the RI (reflection index) value, which is a measure of the stiffness of the arteries, was higher after eating a salty meal. In our study, both groups ( $\mathrm{HC}$ and $\mathrm{LC}$ ) had similar RI values.

The present findings also showed higher levels of ALT and LDL-C in the red meat consumption group. LDL-C concentration correlates positively with body weight and is a risk factor for non-alcoholic fatty liver disease (NAFLD) (Vernon et al., 2011).

Greater red meat intake may have negative effects on liver function (Montonen et al., 2013). A randomised study by Bergeron et al. (2019) provided interesting information that both white meat and red meat cause a comparable increase in LDL-C cholesterol. Furthermore, the increase of this type of lipoprotein was more related to the large LDL-C (Ib LDL) with high atherogenic potential than the high density LDL-C (dsLDL) (Bergeron et al., 2019). The results of 36 randomised controlled studies assessing the effects of red meat in various diets on cardiovascular risk factors were inconclusive. Despite the exclusion of red meat, no significant effects on metabolic parameters (LDL-C, HDL-C, total cholesterol, ApoA1, ApoB) were found (Oh et al., 2015). The surprising results of the study are explained by the beneficial effects of a properly selected diet. In addition, it is suggested that replacing red meat with high-quality vegetable protein has hypolipomising potential. Similar conclusions suggesting the small impact of reducing the consumption of red meat on the main cardiometabolic factors, as well as mortality and the incidence of cancer, were issued by a team of experts from Nutritional Recommendations (NutriRECS) (Johnston et al., 2018). Our results demonstrate that populations who consumed whole grain products more often had lower levels of $\mathrm{Hb}$, Creat, and ALT and higher concentrations of CRP. Other authors have found negative correlations between CRP and whole grain products (Brownlee et al., 2010; de Mello et al., 2011; Lefevre and Jonnalagadda, 2012; Mann et al., 2015; Montonen et al., 2013; Tighe et al., 2010). $\mathrm{Xu}$ et al. (2018) analysed nine randomised control trials that assessed the effects of wholegrain products on inflammatory markers (CRP, IL6, TNF- $\alpha$, interleukin$1 \beta$ ), obtaining a significant decrease in CRP as a result of the diet. The study by Jensen et al. (2006) also showed the beneficial effects of whole grains on glucose profile and lipid profile, without affecting the concentration of CRP.

Confirmation of our results thus requires further research. The lower $\mathrm{Hb}$ levels in the consumption group may be a consequence of higher fibre intake with wholegrain products, which may inhibit absorption of iron in the bowels (Staffolo et al., 2012). The relationship between wholegrain product consumption and lower levels of Creat and ALT may be partly explained by the beneficial effects of these products on the kidneys and liver function (Garcin et al., 2008; Noyez et al., 2006).

Findings have shown the beneficial effects of whole grains on kidney function, showing lower creatinine levels in people using these foods in their diet. Diet therapy is an important element of comprehensive therapy in patients with chronic kidney disease. However, KIDGO guidelines (Clinical Practice Guideline for Acute Kidney Injury) still do not apply to the consumption of whole grains and/or dietary fibre in patients with renal failure.

The beneficial effects of dietary fibre contained in whole grains on the biochemical parameters of the kidneys (creatinine, urea) has been recognised. Moreover, the beneficial effects of antioxidants contained in bran and cereal germs, which include B vitamins, vitamin $\mathrm{E}$, selenium, and zinc, have been recognised. These compounds, either directly or as part of antioxidant enzymes, reduce oxidative stress and, in a further response to inflammatory processes, prevent the impact of free oxygen radicals on cellular structures. A wellknown example is the consequence of the oxidation of low-density lipoprotein (oxLDL, oxidised LDL), which becomes more atherogenic, increasing the risk of atherosclerosis and coronary heart disease. The effects of chronic low-grade inflammation (LGI) and oxidative stress on the development of chronic diseases has been reported many times (Steven et al., 2019).

The effects of whole grains on liver function parameters have also been proven. In the study by Montonen et al. (2013), lower levels of GGTP, ALT, and hs-CRP were found. In the context of these analyses, hepatic enzymes can be considered potential non-specific markers of oxidative stress. Our observations underline the hypothesis that dietary factors can modulate 
markers, which may be potential mediators associated with cardiometabolic risk factors. The high intake of whole grains can provide protection by improving insulin sensitivity, lowering insulin and glucose levels, and inhibiting inflammation and oxidative stress.

Our results also demonstrate that eating wholegrain products is not associated with hyperlipidaemia, atherosclerosis, hypertension, or type-2 diabetes mellitus. The prevalence of cardiovascular disorders may be connected with higher concentrations of CRP as an inflammation marker, which may partly explain our results. Some authors have shown that consuming wholegrain products more often may lower the risk of metabolic disorders (Huang et al., 2015; Tighe et al., 2010).

Consumption of dark chocolate can reduce PPT (peak to peak time), which is the time that elapses from the onset of the first systolic and second diastolic peak of the pulse wave, resulting in increased vascular resistance and increased risk of hypertension (Skrypnik et al., 2020). Our results have shown that populations consuming dark chocolate had reduced PPT, which correlates with the PTT (pulse transit time) pulse wave propagation speed and reflects vessel stiffness.

We have also shown that populations who consume more dark chocolate have higher levels of UA. Higher levels of UA may be a consequence of the greater supply of purines in dark chocolate. Uric acid is the final product of the catabolism of purines (Staffolo et al., 2012). According to many authors, a higher UA concentration is positively associated with metabolic disorders and may be a marker of the risk of developing cardiovascular disease and type- 2 diabetes. An increased concentration of uric acid by $1 \mathrm{mg} / \mathrm{dL}$ above the norm increases the risk of total mortality by as much as $39 \%$ (Borghi et al., 2018). In this research, dark chocolate eaters had a markedly lower PPT, which indicates stiffness of the arteries. The development of hypertension associated with elevated uric acid results from several mechanisms, which include oxidative stress and inflammation, endothelial dysfunction, proliferation of the smooth muscle of the renal vessels, and activation of the renin-angiotensin-aldosterone system.

However, it should be noted that dark chocolate has higher flavonoid content than red wine, black tea, or cranberry juice (Steinberg et al., 2003). In other studies, the beneficial effects of the consumption of polyphenol-rich dark chocolate on lowering glucose, cholesterol levels, and blood pressure have been demonstrated (Al-Dujaili et al., 2015; Mellor et al., 2010), confirming the hypothesis that small doses of dark chocolate may have a positive impact on suppressing metabolic syndrome.

\section{LIMITATIONS}

Our study has several limitations. Our population was rather small, which may have affected the results. The population age range was broad (18-70 years), and different age brackets might be expected to present different findings. Moreover, we did not use 24-hour recall to assess dietary intake, and our results were based only on a food frequency questionnaire. We did not investigate the contents of the breakfasts, so we could not analyze the influence of particular ingredients on the studied parameters.

\section{CONCLUSION}

The results of our study show that the investigated dietary parameters have a significant impact on serum lipid concentrations, serum biochemical parameters of liver and renal function and inflammation, whole blood morphology, and arterial stiffness in a population of the Greater Poland (Wielkopolska) region. Regular consumption of red meat and dark chocolate intensifies CV risk in the Greater Poland (Wielkopolska) population, contrary to the consumption of whole grain products.

\section{REFERENCES}

Al-Dujaili, E. A. S., Farhat, G., Drummond, S., Fyfe, L., McDougall, G. (2015). Comparison of the effects of high versus low-polyphenol dark chocolate on body weight and biochemical markers: A randomized trial. Retrieved from https://eresearch.qmu.ac.uk/handle/20.500.12289/4065

Alaei-Shahmiri, F., Zhao, Y., Sherriff, J. (2015). Assessment of vascular function in individuals with hyperglycemia: A cross-sectional study of glucose - Induced changes in digital volume pulse. J. Diab. Metab. Dis., 14(1). https:// doi.org/10.1186/s40200-015-0153-2 
Skrypnik, K., Bogdanski, P., Kubasik, M., Wawrzyniak, N., Markuszewski, L., Suliburska, J. (2020). The influence of dietary patterns on arterial stiffness, lipid metabolism, and liver and renal function in the population of Greater Poland. Acta Sci. Pol. Technol. Aliment., 19(3), 301-318. http://dx.doi.org/10.17306/J.AFS.2020.0848

Atanassova, B. D., Tzatchev, K. N. (n.d.). Ascorbic acid important for iron metabolism. Folia Med., 50(4), 11-16. Retrieved from http://www.ncbi.nlm.nih.gov/pubmed/ 19209525

Bergeron, N., Chiu, S., Williams, P. T., King, M. S., Krauss, R. M. (2019). Effects of red meat, white meat, and nonmeat protein sources on atherogenic lipoprotein measures in the context of low compared with high saturated fat intake: a randomized controlled trial. Am. J. Clin. Nutr., 110(1), 24-33. https://doi.org/10.1093/ajen/ nqz035

Borghi, C., Tykarski, A., Widecka, K., Filipiak, K. J., Domienik-Karłowicz, J., Kostka-Jeziorny, K., ..., Mancia, G. (2018). Expert consensus for the diagnosis and treatment of patient with hyperuricemia and high cardiovascular risk. Cardiol. J., 25(5), 545-564. https://doi. org/10.5603/CJ.2018.0116

Brownlee, I. A., Moore, C., Chatfield, M., Richardson, D. P., Ashby, P., Kuznesof, S. A., ..., Seal, C. J. (2010). Markers of cardiovascular risk are not changed by increased whole-grain intake: the WHOLEheart study, a randomised, controlled dietary intervention. Brit. J. Nutr., 104(1), 125-134. https://doi.org/10.1017/ S0007114510000644

Lalonde, M. (1974). A new perspective on the health of Canadians. Ottawa, ON: Minister of Supply and Services Canada. Retrieved from: http://www.phac-aspc.gc.ca/ ph-sp/pdf/perspect-eng.pdf

Cocate, P. G., Natali, A. J., Oliveira, A. de, Alfenas, R. de C., Peluzio, M. do C. G., Longo, G. Z., ..., Hermsdorff, H. H. M. (2015). Red but not white meat consumption is associated with metabolic syndrome, insulin resistance and lipid peroxidation in Brazilian middle-aged men. Eur. J. Prev. Card., 22(2), 223-230. https://doi. org/10.1177/2047487313507684

de Mello, V. D. F., Schwab, U., Kolehmainen, M., Koenig, W., Siloaho, M., Poutanen, K., ..., Uusitupa, M. (2011). A diet high in fatty fish, bilberries and wholegrain products improves markers of endothelial function and inflammation in individuals with impaired glucose metabolism in a randomised controlled trial: The Sysdimet study. Diabetologia, 54(11), 2755-2767. https://doi. org/10.1007/s00125-011-2285-3

Staffolo, D. M., Bevilacqua, E. A., Susana, M., Albertengo, L. (2012b). Dietary fiber and availability of nutrients: A case study on yoghurt as a food model. In D. N. Karunarate (Ed.), The complex world of polysaccharides. InTechOpen. https://doi.org/10.5772/54031

Friedewald, W. T., Levy, R. I., Fredrickson, D. S. (1972). Estimation of the concentration of low-density lipoprotein cholesterol in plasma, without use of the preparative ultracentrifuge. Clin. Chem., 18(6), 499-502.

Garcin, J. M., Bronstein, J. A., Cremades, S., Courbin, P., Cointet, F. (2008). Acute liver failure is frequent during heat stroke. World J. Gastroenter., 14(1), 158-159. https://doi.org/10.3748/WJG.14.158

Giboney, P. T. (2005). Mildly elevated liver transaminase levels in the asymptomatic patient. Am. Fam. Physician, 71(6), 1105-10. Retrieved from http://www.ncbi.nlm. nih.gov/pubmed/15791889

Gomes, P. M., Sá, R. W. M., Aguiar, G. L., Paes, M. H. S., Alzamora, A. C., Lima, W. G., ..., Cardoso, L. M. (2017). Chronic high-sodium diet intake after weaning lead to neurogenic hypertension in adult Wistar rats. Sci. Rep., 7(1), 5655. https://doi.org/10.1038/s41598-01705984-9

Huang, T., Xu, M., Lee, A., Cho, S., Qi, L. (2015). Consumption of whole grains and cereal fiber and total and cause-specific mortality: prospective analysis of 367,442 individuals. BMC Med., 13(1), 59. https://doi. org/10.1186/s12916-015-0294-7

Jensen, M. K., Koh-Banerjee, P., Franz, M., Sampson, L., Grønbæk, M., Rimm, E. B. (2006). Whole grains, bran, and germ in relation to homocysteine and markers of glycemic control, lipids, and inflammation. Am. J. Clin. Nutr., 83(2), 275-283. https://doi.org/10.1093/ ajen/83.2.275

Johnson-Wimbley, T. D., Graham, D. Y. (2011). Diagnosis and management of iron deficiency anemia in the $21 \mathrm{st}$ century. Ther. Adv. Gastroenter., 4(3), 177-184. https:// doi.org/10.1177/1756283X11398736

Johnston, B. C., Alonso-Coello, P., Bala, M. M., Zeraatkar, D., Rabassa, M., Valli, C., ..., Guyatt, G. H. (2018). Methods for trustworthy nutritional recommendations NutriRECS (Nutritional Recommendations and accessible Evidence summaries Composed of Systematic reviews): A protocol. BMC Med. Res. Methodol., 18(1). https://doi.org/10.1186/s12874-018-0621-8

Kant, A. K., Andon, M. B., Angelopoulos, T. J., Rippe, J. M. (2008). Association of breakfast energy density with diet quality and body mass index in American adults: National Health and Nutrition Examination Surveys, 1999-2004. Am. J. Clin. Nutr., 88(5), 1396-404. https:// doi.org/10.3945/ajen.2008.26171

Larsson, S. C., Virtamo, J., Wolk, A. (2011). Red meat consumption and risk of stroke in Swedish women. Stroke, 42(2), 324-329. http://dx.doi.org/10.1161/STROKEAHA. 110.596510

Lee, T. S., Kim, J. S., Hwang, Y. J., Park, Y. C. (2016). Habit of eating breakfast is associated with a lower risk of 
Skrypnik, K., Bogdanski, P., Kubasik, M., Wawrzyniak, N., Markuszewski, L., Suliburska, J. (2020). The influence of dietary patterns on arterial stiffness, lipid metabolism, and liver and renal function in the population of Greater Poland. Acta Sci. Pol. Technol. Aliment., 19(3), 301-318. http://dx.doi.org/10.17306/J.AFS.2020.0848

hypertension. J. Lifestyle Med., 6(2), 64-67. https://doi. org/10.15280/jlm.2016.6.2.64

Lefevre, M., Jonnalagadda, S. (2012). Effect of whole grains on markers of subclinical inflammation. Nutr. Rev., 70(7), 387-396. https://doi.org/10.1111/j.17534887.2012.00487.x

Mann, K. D., Pearce, M. S., McKevith, B., Thielecke, F., Seal, C. J. (2015). Whole grain intake and its association with intakes of other foods, nutrients and markers of health in the National Diet and Nutrition Survey rolling programme 2008-11. Brit. J. Nutr., 113(10), 1595-602. https://doi.org/10.1017/S0007114515000525

Meksawan, K., Pongthananikorn, S., Tongyonk, L. (2014). Breakfast skipping is a risk factor for metabolic syndrome (641.2). FASEB J., 04(28), 1. Retrieved from https://www.fasebj.org/doi/abs/10.1096/fasebj.28.1 supplement.641.2

Mellor, D. D., Sathyapalan, T., Kilpatrick, E. S., Beckett, S., Atkin, S. L. (2010). High-cocoa polyphenol-rich chocolate improves HDL cholesterol in Type 2 diabetes patients. Diabet. Med., 27(11), 1318-21. Retrieved from http://www.ncbi.nlm.nih.gov/pubmed/20968113

Millasseau, S. C., Ritter, J. M., Takazawa, K., Chowienczyk, P. J. (2006). Contour analysis of the photoplethysmographic pulse measured at the finger. J. Hypert., 24, 8, 1449-1456. https://doi.org/10.1097/01. hjh.0000239277.05068.87

Min, C., Noh, H., Kang, Y.-S., Sim, H. J., Baik, H. W., Song, W. O., ..., Joung, H. (2011). Skipping breakfast is associated with diet quality and metabolic syndrome risk factors of adults. Nutr. Res. Pract., 5(5), 455-463. https://doi.org/10.4162/nrp.2011.5.5.455

Montonen, J., Boeing, H., Fritsche, A., Schleicher, E., Joost, H.-G., Schulze, M. B., ..., Pischon, T. (2013). Consumption of red meat and whole-grain bread in relation to biomarkers of obesity, inflammation, glucose metabolism and oxidative stress. Eur. J. Nutr., 52(1), 337-345. https://doi.org/10.1007/s00394-012-0340-6

National Kidney Foundation. (2002). K/DOQI clinical practice guidelines for chronic kidney disease: evaluation, classification, and stratification. Am. J. Kidney Dis., 39(2 Suppl 1), S1-266. Retrieved from http://www.ncbi. nlm.nih.gov/pubmed/11904577

Noyez, L., Plesiewicz, I., Verheugt, F. W. A. (2006). Estimated creatinine clearance instead of plasma creatinine level as prognostic test for postoperative renal function in patients undergoing coronary artery bypass surgery. Eur. J. Cardio-Thoracic Surgery, 29(4), 461-465. https:// doi.org/10.1016/j.ejcts.2006.01.024
Oh, S. W., Han, K. H., Han, S. Y., Koo, H. S., Kim, S., Chin, H. J. (2015). Association of sodium excretion with metabolic syndrome, insulin resistance, and body fat. Medicine, 94(39), e1650. https://doi.org/10.1097/ MD.0000000000001650

McKeown, P., Noad, R., McCall, D., McKinley, M., Woodside, J. (2014). Effect of a polyphenol-rich diet on vascular function and other markers of cardiovascular risk. Heart, 100, A62.

Skrypnik, D., Mostowska, A., Jagodziński, P. P., Bogdański, P. (2020). Association of rs699947 (-2578 C/A) and rs2010963 (-634 G/C) single nucleotide polymorphisms of the VEGF Gene, VEGF-A and leptin serum level, and cardiovascular risk in patients with excess body mass: A case-control study. J. Clin. Med., 9(2), 469. https:// doi.org/10.3390/jcm9020469

Steinberg, F. M., Bearden, M. M., Keen, C. L. (2003). Cocoa and chocolate flavonoids: Implications for cardiovascular health. J. Am. Diet. Assoc., 103(2), 215-223. https://doi.org/10.1053/jada.2003.50028

Stepien, M., Kujawska-Luczak, M., Szulinska, M., Kregielska-Narozna, M., Skrypnik, D., Suliburska, J., ... Bogdanski, P. (2018). Beneficial dose-independent influence of Camellia sinensis supplementation on lipid profile, glycemia, and insulin resistance in an $\mathrm{NaCl}$-induced hypertensive rat model. J. Physiol. Pharmacol., 69(2). https://doi.org/10.26402/jpp.2018.2.13

Steven, S., Frenis, K., Oelze, M., Kalinovic, S., Kuntic, M., Jimenez, M. T. B., ..., Daiber, A. (2019). Vascular inflammation and oxidative stress: Major triggers for cardiovascular disease. Oxidat. Med. Cell. Long. ID 7092151. https://doi.org/10.1155/2019/7092151

Szulińska, M., Stępień, M., Kręgielska-Narożna, M., Suliburska, J., Skrypnik, D., Bąk-Sosnowska, M., ..., Bogdański, P. (2017). Effects of green tea supplementation on inflammation markers, antioxidant status and blood pressure in $\mathrm{NaCl}$-induced hypertensive rat model. Food Nutr. Res., 61(1), 1295525. https://doi.org/10.108 0/16546628.2017.1295525

Tighe, P., Duthie, G., Vaughan, N., Brittenden, J., Simpson, W. G., Duthie, S., ..., Thies, F. (2010). Effect of increased consumption of whole-grain foods on blood pressure and other cardiovascular risk markers in healthy middle-aged persons: a randomized controlled trial. Am. J. Clin. Nutr., 92(4), 733-740. https://doi.org/10.3945/ ajen.2010.29417

Tokede, O. A., Gaziano, J. M., Djoussé, L. (2011). Effects of cocoa products/dark chocolate on serum lipids: a metaanalysis. Eur. J. Clin. Nutr., 65(8), 879-886. https://doi. org/10.1038/ejen.2011.64 
Trzeciak, B., Gutknecht, P., Molisz, A., Siebert, J. (2017). Fatal cardiovascular risk in Poland as determined via internet. Ann. Agric. Environ. Med., 24(2), 176-180. https://doi.org/10.5604/12321966.1235161

Vernon, G., Baranova, A., Younossi, Z. M. (2011). Systematic review: The epidemiology and natural history of non-alcoholic fatty liver disease and non-alcoholic steatohepatitis in adults. Alim. Pharm. Therap., 34(3), 274 285. https://doi.org/10.1111/j.1365-2036.2011.04724.x

Walkowska, A., Kuczeriszka, M., Sadowski, J., Olszyñski, K. H., Dobrowolski, L., Červenka, L., ..., Kompanow-
ska-Jezierska, E. (2015). High salt intake increases blood pressure in normal rats: Putative role of 20-HETE and no evidence on changes in renal vascular reactivity. Kidney Blood Press. Res., 40(3), 323-334. https://doi. org/10.1159/000368508

Xu, Y., Wan, Q., Feng, J., Du, L., Li, K., Zhou, Y. (2018). Whole grain diet reduces systemic inflammation: A meta-analysis of 9 randomized trials. Medicine, 97(43). https://doi.org/10.1097/MD.0000000000012995 